\section{P53 BIRTH AND CHILDHOOD FACTORS AND LATE LIFE CEREBROVASCULAR DISEASE: AN ANALYSIS OF 3 LONGITUDINAL COHORT STUDIES}

${ }^{1}$ EV Backhouse*, ${ }^{2}$ SD Shenkin, ${ }^{1} \mathrm{~A}$ McIntosh, ${ }^{3}$ I Deary, ${ }^{1} \mathrm{M}$ Bastin, ${ }^{4} \mathrm{~S}$ de Rooij, ${ }^{4} \mathrm{~T}$ Roseboom, ${ }^{1} \mathrm{JM}$ Wardlaw. ${ }^{1}$ Centre for Clinical Brain Sciences, University of Edinburgh, Edinburgh, UK ${ }^{2}$ Geriatric Medicine, University of Edinburgh, Edinburgh, UK; ${ }^{3}$ Centre for Cognitive Ageing and Cognitive Epidemiology, University of Edinburgh, Edinburgh, UK; ${ }^{4}$ Center for Repoductive Medicine, University of Amsterdam, Amsterdam, The Netherlands

10.1136/jech-2017-SSMAbstracts.154

Background Cerebral small vessel disease (cSVD) is a major cause of stroke and dementia. Midlife vascular disease and adult socioeconomic status (SES) are established risk factors. Less is known about the effect of factors earlier in life. A recent meta-analysis found that lower levels of childhood IQ, childhood SES and education increased the risk of CSVD in later life but was unclear if these relationships persist after adjustment for vascular risk factors or adult SES.

Methods We analysed birth parameters including birth and placental weight (grams), measures of childhood SES such as father's occupation (manual/non-manual), toilet (outdoors; number of people sharing), childhood IQ and premorbid adult IQ using the National Adult Reading Test (NART) and education (years) from participants from 3 cohort studies: the Dutch famine birth cohort $(\mathrm{n}=118)$, the Lothian Birth Cohort $1936(\mathrm{LBC1936,n=685)}$ and the Simpson cohort $(\mathrm{n}=110)$. We analysed CSVD features individually, then summed into a total "cSVD score" (range 1-4) and imaging evidence of infarcts. Data were adjusted for vascular risk factors and adult SES, analysed separately for each cohort and meta-analysed, adjusted for vascular risk factors and adult SES.

Results Across the 3 cohorts increasing birth weight was associated with lower cSVD score (OR 0.999 95\% CI 0.998$0.999, \mathrm{p}=0.02)$. In the Simpson cohort increasing placental weight was associated lower cSVD score (OR 0.995 95\% CI $0.991-0.999, \mathrm{p}=0.01$ ), fewer white matter hyperintensities (WMH) (OR 0.995 95\% CI 0.99-0.999), cerebral microbleeds (CMBs) (OR 0.995 95\% CI 0.991-0.999,, $\mathrm{p}=0.01$ ) and infarcts (OR $0.9995 \%$ CI $0.98-0.998, \mathrm{p}=0.02$ ). Higher childhood IQ was associated with fewer lacunes (all studies; OR 0.98 95\% CI 0.97-0.99 $\mathrm{p}=0.04$ ) and infarcts (LBC36; OR 0.99 $95 \%$ CI $0.97-0.99, \mathrm{p}=0.04$ ). Higher NART score was associated with fewer infarcts (all studies; OR 0.97 95\% CI $0.95-$ $0.99, \mathrm{p}<0.01)$. Having an outdoor toilet and more people sharing a toilet in childhood were associated with more infarcts (Simpson cohort; OR 13.31 95\% CI 1.52-116.38, $\mathrm{p}=0.02$; OR $1.1895 \%$ CI 1.001-1.4 p=0.049). Lower education was associated with more CMBs (all studies; OR 1.78 95\% CI $1.04-3.04, \mathrm{p}=0.04)$.

Discussion Birth parameters including birth and placental weight may influence risk of CSVD in later life. Childhood factors such as IQ, education and SES may influence some CSVD features and risk of infarcts but it was not possible in these data to determine whether they contribute independently to WMH or total cSVD or not. The effects sizes and potential impact of these findings suggest that larger samples are needed to robustly test these associations.

\section{P54 CHANGES IN PHYSICAL AND MENTAL HEALTH FUNCTIONING DURING RETIREMENT TRANSITION: A REGISTER-LINKAGE FOLLOW-UP STUDY}

${ }^{1} \mathrm{M}$ Manty ${ }^{*},{ }^{2,3,4} \mathrm{~A}$ Kouvonen, ${ }^{1,5} \mathrm{~T}$ Lallukka, ${ }^{1} \mathrm{~J}$ Lahti, ${ }^{1} \mathrm{E}$ Lahelma, ${ }^{1} \mathrm{O}$ Rahkonen. ${ }^{1}$ Department of Public Health, University of Helsinki, Helsinki, Finland; ${ }^{2}$ Department of Social Research, University of Helsinki, Helsinki, Finland; ${ }^{3}$ SWPS University of Social Sciences and Humanities in Wroclaw, Wroclaw, Poland; ${ }^{4}$ Centre for Public Health, Queen's University Belfast, Belfast, UK; ${ }^{5}$ Finnish Institute of Occupational Health, Helsinki, Finland

\subsection{6/jech-2017-SSMAbstracts. 155}

Background The impact of retirement on health-related functioning, an important indicator of individual's ability to function in everyday life, is poorly understood. Thus the aim of this study was to examine the association between transition to retirement, and changes in physical and mental health functioning among Finnish municipal employees.

Methods Follow-upsurvey data were collected among ageing employees of the City of Helsinki, Finland, at three Phases: 1 (2000-2002), 2 (2007) and 3 (2012). Physical and mental health functioning were measured using the Short-Form 36 questionnaire at each Phase. Retirees between Phases 1 and 3 were identified from the national registers of the Finnish Centre for Pensions: Full-time statutory retirement $(n=1464)$, part-time retirement not due to health reasons $(n=404)$ and disability retirement due to health reasons $(n=462)$. Generalised estimating equations were used to study the associations between transition to retirement and changes in health functioning. We also examined whether changes in functioning vary by gender, occupational status, or different health- and life-style factors.

Results Statutory and part-time retirement were not associated with changes in physical health functioning during retirement transition process when adjusting for gender and age $(\beta 0.1$, $95 \% \mathrm{CI}-0.3$ to 0.5 and $-1.0,-1.8$ to 0.1 , respectively), whereas clear decline was observed among disability retirees $(-4.3,-5.4$ to -3.2$)$. Higher occupational class before retirement and sedentary lifestyle were associated with greater decline in physical health functioning. Mental health functioning improved during the retirement transition process among statutory and part-time retirees $(1.9,1.4$ to 2.4 and $2.0,1.0$ to 3.0 , respectively) while no association was observed among disability retirees $(-0.3,-1.7$ to 1.0$)$.

Conclusion Disability retirement was associated with decrease in physical health functioning, and statutory retirement with a slight improvement in mental health functioning. Evidence on changes in physical and mental functioning during retirement transition process may provide useful information for interventions to promote healthy ageing.

\section{P55 PAIN AND ROUTES OF EXIT OUT OF PAID EMPLOYMENT AMONG BRITISH CIVIL SERVANTS: A FOLLOW-UP STUDY 1985-2013}

${ }^{1,2} \mathrm{TL}$ Lallukka*, ${ }^{2} \mathrm{MM}$ Mänty, ${ }^{3} \mathrm{CC}$ Cooper, ${ }^{4} \mathrm{MF}$ Fleischmann, ${ }^{5,6,7}$ AK Kouvonen, ${ }^{4} \mathrm{JH}$ Head, ${ }^{1} \mathrm{JIH}$ Halonen. 'Finnish Institute of Occupational Health, Helsinki, Finland; ${ }^{2}$ Department of Public Health, University of Helsinki, Helsinki, Finland; ${ }^{3}$ MRC Lifecourse Epidemiology Unit, Universities of Southampton and Oxford, UK; ${ }^{4}$ Institute of Epidemiology and Health, University College London, London, UK; ${ }^{5}$ Department of Social Research, University of Helsinki, Helsinki, Finland; ${ }^{6}$ SWPS University of Social Sciences and Humanities in Wroclaw, Wroclaw, Poland; ' ${ }^{7}$ dministrative Data Research Centre-Northern Ireland, Centre for Public Health, Queen's University Belfast, Belfast, UK

10.1136/jech-2017-SSMAbstracts. 156 
Background Pain is a risk factor for work disability, however, routes of exit out of paid employment have not been examined in detail. Thus, the aim of this study was to examine the contribution of repeated exposure to pain and subsequent transitions out of paid employment. A further aim was to examine, whether the associations vary by sex, age, occupational class, job demands, job control and body mass index.

Methods We included participants of the Whitehall II study cohort ( $n=8445,69 \%$ men, aged 35-55 at baseline), who had provided at least 2 measurements of back pain between phases 1 and 3 (1985-1994). People with pain at 1 point in time, and pain at 2-3 time points were compared to people with no pain at any phase. Exit from paid employment was observed between 1995-2013 (phases 4-11). Routes of exit were 1) health-related (long-term sick or retirement on health grounds), 2) unemployment, 3) other early exit, 4) retirement not related to health. Those remaining working served as a reference group. Sex, age, parental and own socioeconomic position, job demands, job control, and body mass index were controlled for. Repeated measures logistic regression models were fitted.

Results Altogether 10\% exited the employment due to healthrelated reasons, $2 \%$ due to unemployment and $6.5 \%$ due to other reasons. Pain contributed to the transitions out of paid employment due to health reasons. After full adjustments, reporting pain at one time point $(26 \%)$ was not associated with exit due to health reasons, while reporting repeated pain was associated with such exit (18\%, OR 1.52, 95\% CI 1.162.00), as compared to those who did not report pain during phases 1-3 (56\%). Associations were somewhat stronger among middle or lower class employees and non-existent among high class employees, but otherwise differences e.g. by age, working conditions or obesity were small. The risk of exit due to other routes than health-related did not vary between people with or without pain.

Conclusion These results highlight the need for early detection of repeated pain, to prevent the risk of health-related early exit out of paid employment. As the risk varies between different occupational groups and somewhat by working conditions, this emphasises the importance of identification of high risk groups and their modifiable risk factors, such as working conditions.

\section{P56 DO WORKING CONDITIONS ALTER TO ACCOMMODATE OLDER WORKERS' CHANGING NEEDS WHEN THEIR HEALTH DECLINES: A 10-YEAR FOLLOW-UP OF THE ENGLISH LONGITUDINAL STUDY OF AGEING}

MS Fleischmann*, J Head. Epidemiology and Public Health, University College London, London, UK

\subsection{6/jech-2017-SSMAbstracts. 157}

Background Due to transformations in pension policies, older workers, even those with poor health, are expected to extend their working lives. In this context, favourable working conditions are frequently discussed as modifiable characteristics that might help to their extended working lives. We investigate whether working conditions actually change if older workers' physical or mental health is declining.

Methods Data on older men and women, aged 50-70 and observed at least twice, from six waves of the English Longitudinal Study of Ageing (ELSA) were used $(n=2,958)$. We observed both the onset of a chronic disease, i.e. diagnose/ incident of diabetes, arthritis, stroke, heart problem, asthma, high blood pressure, cancer, or lung disease, and changes in mental health (CESD-R scale for depression), as well as changes in working conditions. Working conditions encompassed physical job demands, decision authority, social support, job security, and working hours. We used fixed effects models to analyse whether changes in older persons' health could be related to alterations in working conditions.

Results Preliminary results show that after adjusting for individuals' age, the onset of a chronic disease was related to lower decision authority $(b=-0.16[-0.25,-0.07])$ and lower social support $(b=-0.16[-0.25,-0.08])$. These associations also held after adjusting for individuals' mental health. Furthermore, a decline in mental health were related to more unfavourable working conditions, specifically job security $(b=-0.01$ $[-0.2,-0.004])$, social support $(b=-0.05[-0.07,-0.03])$, and decision authority $(b=-0.04 \quad[-0.07,-0.3])$. The changes in working conditions are rather small but significant. Robustness checks are done to account for possible endogeneity.

Conclusion Working conditions do not seem to alter in order to accommodate older workers' changing needs when their health declines. Rather, older workers with declining mental or physical health report deterioration in their working conditions. Future researchers, policy advisors, and employers might want to consider scrutinising the group of older workers with declining health and their special workplace requirements when discussing possibilities for extended working lives.

\section{P57 PHYSICAL FITNESS OFFSETS COGNITIVE DYSFUNCTION IN DEMENTIA}

${ }^{1} \mathrm{D}$ Cadar*, ${ }^{1} \mathrm{~A}$ Hollamby, ${ }^{2} \mathrm{EJ}$ Davelaar. ${ }^{1}$ Department of Behavioural Science and Health, University College London, London, UK; ${ }^{2}$ Department of Psychological Sciences, Birkbeck College, University of London, UK

\subsection{6/jech-2017-SSMAbstracts. 158}

Background Physical fitness has been associated with improved cognition in older age. We explored the associations between physical fitness and cognitive performance in healthy elderly and in individuals with dementia, taking into account the time since dementia diagnosis.

Methods Thirty elderly with dementia and 40 healthy controls completed a battery of standardised cognitive tests: Mini-Mental State Exam, Verbal Fluency, Prospective and Retrospective Memory Questionnaire, Clock Drawing and California Verbal Learning Test. Participants were grouped into high versus low levels of physical fitness (PF) based on their Physical Fitness Questionnaire scores.

A $2 \times 2$ between-subject multivariate analysis of covariance was used to evaluate the associations between PF levels and cognitive performance, adjusting for age, gender, education, occupation, head injury, internet use, brain training, and past levels of exercise. This was followed by a discriminant analysis to obtain a latent score of global cognitive functioning for each individual.

Results Healthy controls showed higher performance scores than individuals with dementia [Pillai's Trace $=0.61, F(10,49)$ $=7.75, \mathrm{p}<0.001]$. PF was associated with better cognitive performance [Pillai's Trace $=0.32, \mathrm{~F}(10,49)=2.29, \mathrm{p}<0.05$ ] and this interacted with health status [Pillai's Trace $=0.30, \mathrm{~F}$ $(10,49)=2.12, \mathrm{p}<0.05]$. A significant discriminant analysis (Wilks $\lambda=0.15, \quad$ Chi-square $=119.46, \quad \mathrm{df}=30, \quad$ canonical 